\title{
Uplink performance investigations of the service area based beyond 3G system JOINT
}

\author{
Y. Liu ${ }^{1}$, T. Weber ${ }^{1}$, and W. Zirwas ${ }^{2}$ \\ ${ }^{1}$ Research Group for RF Communications, University of Kaiserslautern, P.O. Box 3049, 67653 Kaiserslautern, Germany \\ ${ }^{2}$ Siemens AG, ICM N PG SP RC FR, Sankt-Martinstrasse 76, 81541 München, Germany
}

\begin{abstract}
The joint transmission and detection integrated network (JOINT) is a novel OFDM-based (van Nee and Prasad, 2000) air interface solution for beyond 3G (B3G) mobile radio communications systems (Weber et al., 2002). JOINT aims at eliminating the multiple access interference (MAI) and improving the system capacity by the application of MIMO techniques applied in the service area (SA) based system architecture. In a SA based structure the intra-SA multiple access interference (MAI) can be easily combated by algorithms like, e.g., joint detection (JD) (Klein, 1996; Verdu, 1998) in the uplink. The parameters like, e.g., the SA size, the system load and reuse factor show great impacts on the system performances, in terms of the average bit error rate (BER) and the BER statistics. Spectrum efficiency of JOINT is also investigated based on the simulation results.
\end{abstract}

\section{Introduction}

In general the performance of mobile radio systems is limited by interferences. The joint transmission and detection integrated network (JOINT) is a novel OFDM (van Nee and Prasad, 2000) based air interface solution for beyond 3G (B3G) mobile radio communications systems (Weber et al., 2002). Each service area (SA), the entity of JOINT, is equipped with a central unit (CU), to which the access points (APs) of the SA are connected. In each SA multiple mobile terminals (MTs) are active simultaneously. In a SA based system two basic types of the MAI can be discerned, i.e., the intra-SA MAI and the inter-SA MAI. The intra-SA MAI can be easily combated by algorithms like, e.g., joint detection (JD) (Klein, 1996; Verdu, 1998) in the uplink, while the inter-SA MAI remains to be dealt with.

In order to reduce the computational effort of simulations, in the present paper some simplifications are made, e.g., only a single subcarrier is considered, and the channel is assumed to be perfectly known. In the present paper signals on the considered subcarrier are described by their complex amplitudes and the channel is described by its complex transfer function value at the considered subcarrier.

Correspondence to: Y. Liu

(fliu@ rhrk.uni-kl.de)
Complex quantities are underlined, and vectors and matrices are printed in bold face. $(\cdot)^{*}$ and $(\cdot)^{\mathrm{T}}$ designate the complex conjugation and the transposition, respectively. The element in the $i$-th row and $j$-th column of a matrix is denoted by $[\cdot]_{i, j}$.

\section{Scenario description}

\subsection{Multiple-service-area scenario}

In the present subsection a multi-SA scenario for the performance investigations of the SA based system structure is described.

In contrast to cellular systems, the entity of JOINT is the SA, consisting of several neighboring sub-areas, which are equivalent to the cells in the cellular system. It is assumed that there is one AP located at the center of each sub-area, and that there is at most one active MT randomly distributed in each sub-area. As depicted in Fig. 1, the hexagons represent the sub-areas with radius $R$, and the APs and the MTs are located in the corresponding hexagonal sub-areas. The radius $R$ equal to $250 \mathrm{~m}$ of the hexagonal sub-area is assumed in the following investigations.

The neighboring hexagonal sub-areas with the same pattern form a SA. The number $K_{\mathrm{B}}$ of APs per SA also represents the SA size. The neighboring SAs with different patterns form a cluster of SAs. The frequency resources can be reused among the different clusters of SAs. The size of the cluster of SAs is described by the reuse factor $r$. The SAs with the same pattern represent the co-channel SAs, in which we are interested. It is known that in the case of $K_{\mathrm{B}}$ equal to one, i.e., each SA comprising only a single sub-area, the SA-based system architecture is equivalent to the cellular system architecture. Due to the hexagonal sub-areas, it is found, that, whatever the SAs look like, if one of the APs in one $\mathrm{SA}$ is chosen as the reference AP, the reference APs of the neighboring four co-channel SAs always form a diamond, as depicted in Fig. 1. In other words, the reference APs of the co-channel SAs are located along a parallelogram grid. In the following simulations, instead of a scenario with fixed dimensions, the a scenario with a fixed number $N_{\mathrm{SA}}$ of the co-channel SAs, e.g., $N_{\mathrm{SA}}$ equal to $5 \times 5$, is investigated. 


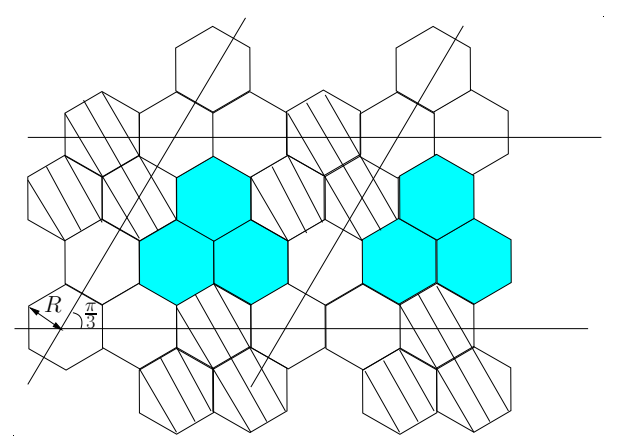

Fig. 1. An example of the SA-based system architecture with the SA size $K_{\mathrm{B}}=3$ and the reuse factor $r=3$.

As shown in Fig. 2, the reference cluster of SAs is at the center of the given scenario. The SA in white in the reference cluster of SAs is the reference SA. The co-channel interfering SAs which have a dominant influence on the reference SA are included so that with the finite scenario we can approximate the ideal infinite scenario.

\subsection{Channel model}

A Rayleigh fading channel model with log-normal fading is applied in the simulations and is discussed in the following. The average path gain between an MT and an AP denoted by $g$ is a $\log$-normally distributed random variable, i.e.,

$g=10^{\frac{G}{10}}$

holds, where $G$ is a normally distributed random variable with standard deviation $\sigma_{\mathrm{G}}$ and mean value $\bar{G}$. $\sigma_{\mathrm{G}}$ takes the value of $4 \mathrm{~dB}$ in the investigations. A dual-slope model (Boejeson et al., 1992; Xia et al., 1992; Steil, 1995) is described in the following. With the break-even-point distance $\rho_{\mathrm{B}}$ (Boejeson et al., 1992; Xia et al., 1992; Steil, 1995), the antenna gains $g_{\mathrm{T}}$ and $g_{\mathrm{R}}$ of the transmit antenna and the receive antenna, respectively, and the wave length $\lambda$, the average logarithmic path gain

$$
\begin{aligned}
& \bar{G}= \\
& \left\{\begin{array}{l}
-10 \alpha_{1} \log _{10}\left(\frac{4 \pi \rho}{\lambda}\right)+10 \log _{10}\left(g_{\mathrm{T}} g_{\mathrm{R}}\right), \\
-10 \alpha_{1} \log _{10}\left(\frac{4 \pi \rho_{\mathrm{B}}}{\lambda}\right)-10 \alpha_{2} \log _{10}\left(\frac{\rho}{\rho_{\mathrm{B}}}\right)+10 \log _{10}\left(g_{\mathrm{T}} g_{\mathrm{R}}\right), \text { for } \rho \geq \rho_{\mathrm{B}}
\end{array}\right.
\end{aligned}
$$

is obtained, where $\alpha_{1} \leq \alpha_{2}$ is assumed. In the investigations, $g_{\mathrm{T}}$ equal to one and $g_{\mathrm{R}}$ equal to one are assumed. The break-even-point distance $\rho_{\mathrm{B}}$ (Boejeson et al., 1992; Xia et al., 1992; Steil, 1995) takes a realistic value of $300 \mathrm{~m}$. The attenuation exponents $\alpha_{1}$ and $\alpha_{2}$ are assumed to be 2.0 and 4.0 , respectively.

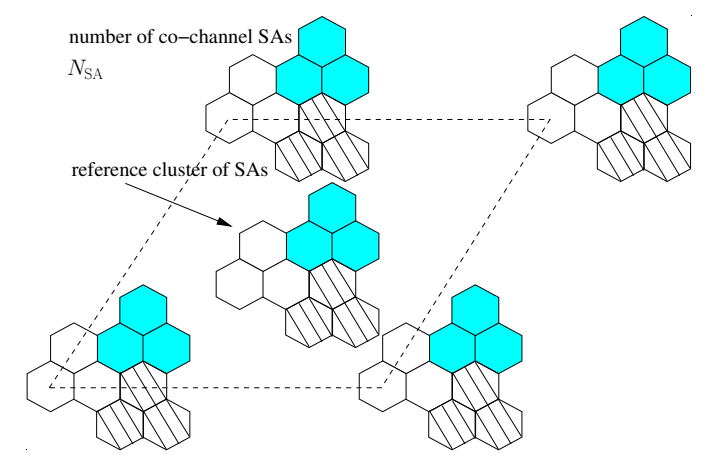

Fig. 2. Considered area with the SA size $K_{\mathrm{B}}=3$ and the reuse factor $r=3$

\section{Subcarrier-wise system model}

In the considered scenario there are in total $N_{\mathrm{SA}}$ co-channel SAs. The total UL data vector

$\underline{\boldsymbol{d}}=\left(\underline{\boldsymbol{d}}^{(1)^{\mathrm{T}}} \ldots \underline{\boldsymbol{d}}^{\left(N_{\mathrm{SA}}\right)^{\mathrm{T}}}\right)^{\mathrm{T}}$

consists of $N_{\mathrm{SA}}$ SA specific data vectors

$\underline{\boldsymbol{d}}^{(i)}=\left(\underline{d}^{((i-1) K+1)} \ldots \underline{d}^{((i-1) K+K)}\right)^{\mathrm{T}}, i=1 \ldots N_{\mathrm{SA}}$,

each of dimension $K$, where $K$ denotes the number of MTs per SA. The elements $\underline{d}^{(i-1) K+k)}$ of $\underline{\boldsymbol{d}}^{(i)}$ of Eq. (4) are assumed to be independent identical distributed with a variance $\sigma_{\mathrm{d}}^{2}$ of real and imaginary parts. For the covariance matrix of the data vector $\underline{\boldsymbol{d}}$ of Eq. (3) follows

$\underline{\mathbf{R}}_{\mathrm{d}}=\mathrm{E}\left\{\underline{\boldsymbol{d}}^{* \mathrm{~T}}\right\}=2 \sigma_{\mathrm{d}}^{2} \mathbf{I}$.

The total channel transfer function (CTF) matrix reads

$\underline{\mathbf{H}}=\left(\begin{array}{ccc}\underline{\mathbf{H}}^{(1,1)} & \cdots & \underline{\mathbf{H}}^{\left(N_{\mathrm{SA}}, 1\right)} \\ \vdots & & \vdots \\ \underline{\mathbf{H}}^{\left(1, N_{\mathrm{SA}}\right)} & \cdots & \underline{\mathbf{H}}^{\left(N_{\mathrm{SA}}, N_{\mathrm{SA}}\right)}\end{array}\right)$

consists of the $N_{\mathrm{SA}} \times N_{\mathrm{SA}}$ sub-matrices

$\underline{\mathbf{H}}^{(i, j)}=$

$\left(\begin{array}{ccc}\underline{H}^{\left((i-1) K+1,(j-1) K_{\mathrm{B}}+1\right)} & \cdots & \underline{H}^{\left((i-1) K+K,(j-1) K_{\mathrm{B}}+1\right)} \\ \vdots & & \vdots \\ \underline{H}^{\left((i-1) K+1,(j-1) K_{\mathrm{B}}+K_{\mathrm{B}}\right)} & \cdots & \underline{H}^{\left((i-1) K+K,(j-1) K_{\mathrm{B}}+K_{\mathrm{B}}\right)}\end{array}\right)$,

The elements $\underline{H}^{\left((i-1) K+k,(j-1) K_{\mathrm{B}}+k_{\mathrm{B}}\right)}$ of Eq. (6) describe the CTFs between the MTs of the SA $i$, and the APs of the SA $j$. The total noise vector

$\underline{\boldsymbol{n}}=\left(\underline{\boldsymbol{n}}^{(1)^{\mathrm{T}}} \ldots \underline{\boldsymbol{n}}^{\left(N_{\mathrm{SA}}\right)^{\mathrm{T}}}\right)^{\mathrm{T}}$

consists of $N_{\mathrm{SA}}$ SA specific noise vectors

$\underline{\boldsymbol{n}}^{(i)}=\left(\underline{n}^{\left((i-1) K_{\mathrm{B}}+1\right)} \cdots \underline{n}^{\left((i-1) K_{\mathrm{B}}+K_{\mathrm{B}}\right)}\right)^{\mathrm{T}}, i=1 \ldots N_{\mathrm{SA}}$, 
each of dimension $K_{\mathrm{B}}$. The elements $\underline{n}^{\left((i-1) K_{\mathrm{B}}+k_{\mathrm{B}}\right)}$ of $\underline{\boldsymbol{n}}^{(i)}$ of (9) are assumed to be independent identical and Gaissian distributed with a variance $\sigma^{2}$ of real and imaginary parts. For the covariance matrix of the noise vector $\underline{\boldsymbol{n}}$ of Eq. (8) follows

$\underline{\mathbf{R}}_{\mathrm{n}}=\mathrm{E}\left\{\underline{\boldsymbol{n}}^{* \mathrm{~T}}\right\}=2 \sigma^{2} \mathbf{I}$.

If a constant transmitted energy $T$ per MT is used, the total received vector

$\underline{\boldsymbol{e}}=\left(\underline{\boldsymbol{e}}^{(1)^{\mathrm{T}}} \cdots \underline{\boldsymbol{e}}^{\left(N_{\mathrm{SA}}\right)^{\mathrm{T}}}\right)^{\mathrm{T}}$

consisting of $N_{\mathrm{SA}} \mathrm{SA}$ specific received vectors

$\underline{\boldsymbol{e}}^{(i)}=\left(\underline{e}^{\left((i-1) K_{\mathrm{B}}+1\right)} \ldots \underline{e}^{\left((i-1) K_{\mathrm{B}}+K_{\mathrm{B}}\right)}\right)^{\mathrm{T}}, i=1 \ldots N_{\mathrm{SA}}$,

each of dimension $K_{\mathrm{B}}$, becomes

$\underline{\boldsymbol{e}}=\frac{\sqrt{T}}{\sigma_{\mathrm{d}}} \underline{\mathbf{H}} \underline{\boldsymbol{d}}+\underline{\boldsymbol{n}}$.

\section{Zero forcing joint detection}

In the SA $i$ the linear zero forcing JD (ZF-JD) (Klein, 1996; Verdu, 1998) is employed at the CU. The biased estimate

$$
\begin{aligned}
\underline{\hat{\boldsymbol{d}}}^{(i)} & =\frac{\sigma_{\mathrm{d}}}{\sqrt{T}}\left(\underline{\mathbf{H}}^{(i, i) * \mathrm{~T}} \underline{\mathbf{H}}^{(i, i)}\right)^{-1} \underline{\mathbf{H}}^{(i, i) * \mathrm{~T}} \underline{\boldsymbol{e}}^{(i)} \\
& =\underline{\boldsymbol{d}}^{(i)}+\sum_{i \neq j} \underbrace{\left(\underline{\mathbf{H}}^{(i, i)} \underline{\mathbf{H}}^{(i, i)}\right)^{-1} \underline{\mathbf{H}}^{(i, i)} \underline{\mathbf{H}}^{(j, i)}}_{\underline{\mathbf{X}}^{(i, j)}} \underline{\boldsymbol{d}}^{(j)} \\
& +\frac{\sigma_{\mathrm{d}}}{\sqrt{T}}\left(\underline{\mathbf{H}}^{(i, i) * \mathrm{~T}} \underline{\mathbf{H}}^{(i, i)}\right)^{-1} \underline{\mathbf{H}}^{(i, i) * \mathrm{~T}} \underline{\boldsymbol{n}}^{(i)}
\end{aligned}
$$

of the data vector $\underline{\boldsymbol{d}}^{(i)}$ of Eq. (4) is obtained. The intra-SA MAI is eliminated. The inter-SA MAI corresponding to the second term of the right side of Eq. (14) and the enhanced noise corresponding to the third term of Eq. (14) result in the data estimation error vector

$$
\begin{aligned}
& \underline{\hat{\mathbf{d}}}^{(i)}-\underline{\boldsymbol{d}}^{(i)}= \\
& \quad \sum_{i \neq j} \underline{\mathbf{X}}^{(i, j)} \underline{\boldsymbol{d}}^{(j)}+\frac{\sigma_{\mathrm{d}}}{\sqrt{T}}\left(\underline{\mathbf{H}}^{(i, i)}{ }^{* \mathrm{~T}} \underline{\mathbf{H}}^{(i, i)}\right)^{-1} \underline{\mathbf{H}}^{(i, i)}{ }^{* \mathrm{~T}} \underline{\boldsymbol{n}}^{(i)} .
\end{aligned}
$$

\section{Simulation results}

\subsection{Noise figure}

In the following only the thermal noise of the receiver is considered. The variance $\sigma^{2}$ of the thermal noise can be described by the noise figure $F$, i.e.,

$\sigma^{2}=\kappa T_{0} B F$

holds (Couch, 1997), where $T_{0}$ is the absolute temperature, e.g., equal to $290 \mathrm{~K}, \kappa$ is Boltzmann's constant equal to $1.38 \times 10^{-23} \mathrm{~J} / \mathrm{K}$, and $B$ is the bandwidth of the considered single subcarrier, equal to $39.063 \mathrm{kHz}$. Therefore, with Eq. (16) the relation

$$
\begin{aligned}
& 10 \log _{10}\left(\frac{T}{\sigma^{2}}\right)=10 \log _{10}\left(\frac{T}{10^{-3} \mathrm{~W}}\right) \\
& -10 \log _{10} F 10 \log _{10}\left(\frac{10^{-3} \mathrm{~W}}{k^{\prime} T_{0} B}\right)=T_{\mathrm{dBm}}-F_{\mathrm{dB}}+\text { const }
\end{aligned}
$$

can be obtained. In the following, the average BERs $\bar{P}_{\mathrm{b}}$ versus the difference $\left(T_{\mathrm{dBm}} / \mathrm{dBm}-F_{\mathrm{dB}} / \mathrm{dB}\right)$, which indicates the relation between the transmitted energy and the noise power, will be investigated. In the following typical values of $T_{\mathrm{dBm}}$ equal to $3 \mathrm{dBm}$ and $F_{\mathrm{dB}}$ equal to $5 \mathrm{~dB}$ are assumed (Couch, 1997).

\subsection{Average bit error rate}

Figure 3 depicts the average BER $\bar{P}_{\mathrm{b}}$ curves of JOINT with different combinations of the number $K$ of MTs per SA, the number $K_{\mathrm{B}}$ of APs per SA and the reuse factor $r$, in the uplink. The $\bar{P}_{\mathrm{b}}$ curves of the equivalent cellular system, which are the curves for the case of $K_{\mathrm{B}}$ equal to one, are plotted with dashed lines as references.

The $\bar{P}_{\mathrm{b}}$ curves converge to error floors, due to the fact that the linear ZF-JD (Klein, 1996; Verdu, 1998) is applied based on the CTF matrix $\underline{\tilde{\mathbf{H}}}^{(i, i)}$ of Eq. (7) of the SA $i$ regardless of the other co-channel SAs $j \neq i$. The intra-SA MAI is eliminated, while the inter-SA MAI in the multiple-SA scenario remains to limit the performance.

As the reuse factor $r$ increases, the average distances between the interferers of the co-channel SAs and the receivers in the reference SA correspondingly increase so that the impact of the inter-SA MAI becomes smaller. Consequently, the system performance, in terms of the average BERs, is greatly improved. It can bee seen in Fig. 3 that for the same number $K$ of MTs per SA and the same reuse factor $r$, a larger SA size $K_{\mathrm{B}}$ results in a better average BER performance in the uplink. Through the comparison of JOINT and the cellular system, it is found that the SA-based JOINT does not show advantages over the conventional cellular system for the small reuse factor $r$ equal to one. However, as the reuse factor $r$ increases, the $\bar{P}_{\mathrm{b}}$ curves of JOINT converge to lower error floors.

\subsection{Bit error rate statistics}

Other than a rough assessment of the average BERs $\bar{P}_{\mathrm{b}}$, the outage probability $P_{\text {out }}$ is a more meaningful quality criterion to evaluate the system performance. The outage probability $P_{\text {out }}$ is the probability that the MT-specific BER $P_{\mathrm{b}}$, which is the BER averaged only over the noise and the fast fading for the corresponding MT, is greater than a given outage threshold, i.e., a given maximum acceptable BER $P_{\max }$. In order to comprehensively evaluate the system of JOINT the 

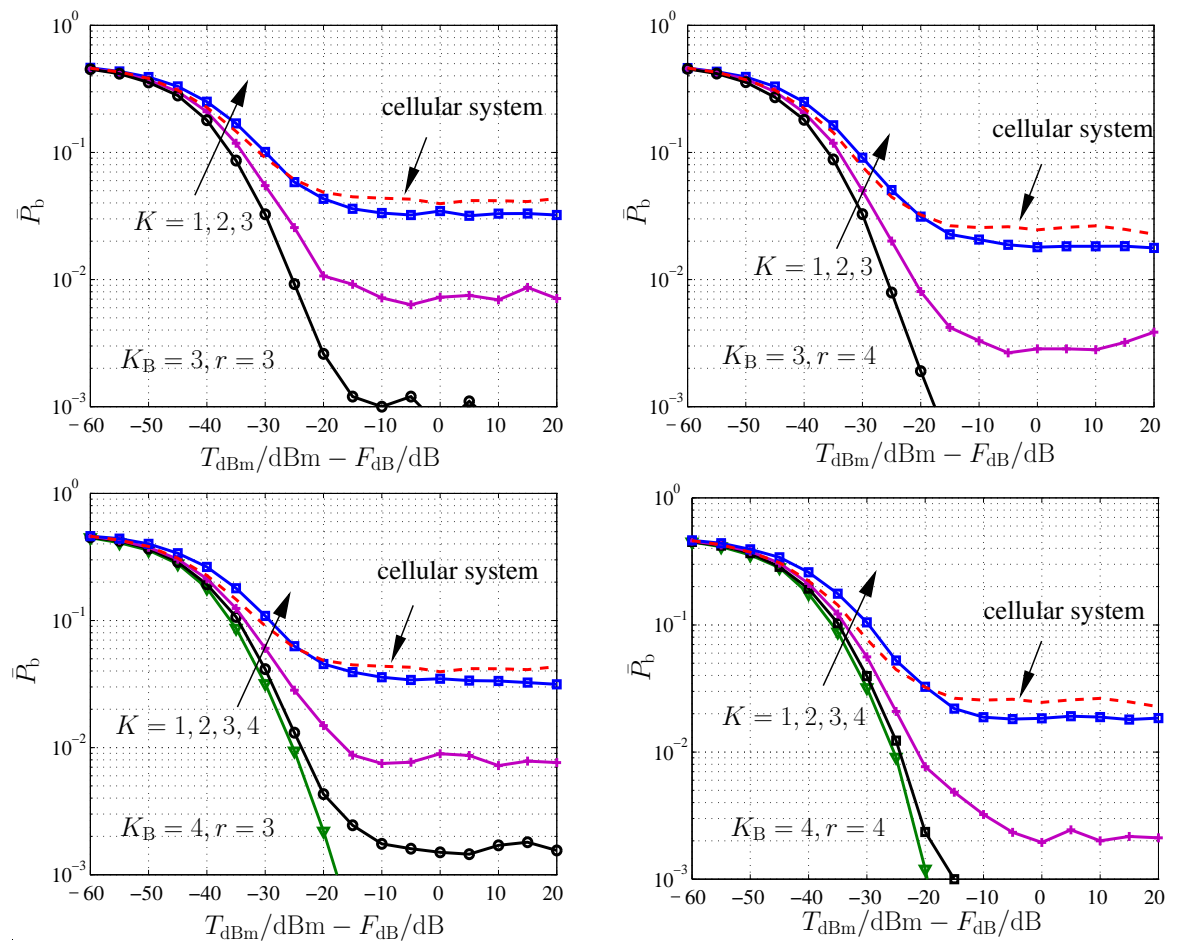

Fig. 3. Average BERs $\bar{P}_{\mathrm{b}}$ in the reference SA in the uplink.
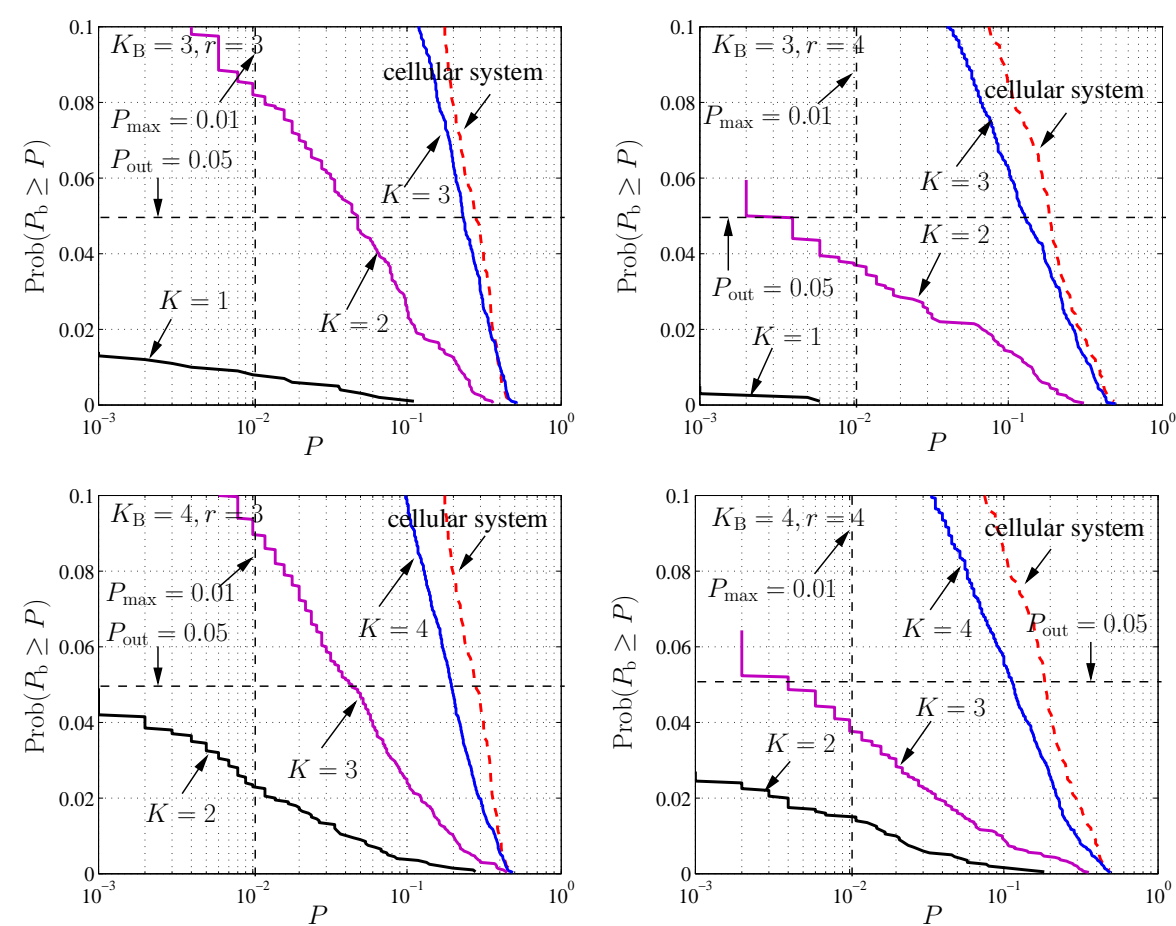

Fig. 4. CCDF curves of the BERs $P_{\mathrm{b}}$ in the uplink. 
Table 1. Spectrum efficiencies $\eta_{\mathrm{c}}$ of the reference system of $K_{\mathrm{B}}=1$ and $K=1$.

\begin{tabular}{ccccccc}
\hline & $r=1$ & $r=3$ & $r=4$ & $r=7$ & $r=9$ & $r=12$ \\
\hline$\frac{\eta_{\mathrm{c}}}{\mathrm{bits} / \mathrm{s} / \mathrm{Hz}}$ & - & - & - & - & - & $\mathbf{0 . 1 3 3 3}$ \\
\hline
\end{tabular}

Table 2. Spectrum efficiencies $\frac{\eta_{\mathrm{c}}}{\text { bits } / \mathrm{s} / \mathrm{Hz}}$ of JOINT.

\begin{tabular}{|c|c|c|c|c|c|c|c|c|c|}
\hline & $r=1$ & $r=3$ & $r=4$ & $r=7$ & & $r=1$ & $r=3$ & $r=4$ & $r=7$ \\
\hline$\frac{K}{K_{\mathrm{B}}}=\frac{1}{3}$ & - & 0.1778 & 0.1333 & 0.0762 & $\frac{K}{K_{\mathrm{B}}}=\frac{1}{4}$ & - & 0.1333 & 0.1 & 0.0571 \\
\hline$\frac{K}{K_{\mathrm{B}}}=\frac{2}{3}$ & - & - & 0.2667 & 0.1524 & $\frac{K}{K_{\mathrm{B}}}=\frac{2}{4}$ & - & 0.2667 & 0.2 & 0.1143 \\
\hline \multirow[t]{2}{*}{$\frac{K}{K_{\mathrm{B}}}=\frac{3}{3}$} & - & - & - & - & $\frac{K}{K_{\mathrm{B}}}=\frac{3}{4}$ & - & - & 0.3 & 0.1714 \\
\hline & & & & & $\frac{K}{K_{\mathrm{B}}}=\frac{4}{4}$ & - & - & - & 0.2286 \\
\hline
\end{tabular}

statistics of the MT-specific BERs $P_{\mathrm{b}}$ in the reference SA are discussed in the following.

Usually for the uncoded transmission, the maximum acceptable BER $P_{\max }$ equal to 0.01 is assumed. The outage probability

$$
P_{\text {out }}=\operatorname{Prob}\left(P_{\mathrm{b}} \geq P_{\max }=0.01\right) \leq 0.05
$$

is chosen as the quality criterion, e.g., for the system with the given values of $T_{\mathrm{dBm}}$ of and $F_{\mathrm{dB}}$. Figure 4 depicts the complementary cumulative distribution function (CCDF) curves of the MT-specific BERs $P_{\mathrm{b}}$ in the reference $\mathrm{SA}$. In each figure, the vertical dashed line indicates the maximum acceptable BER $P_{\max }$, and the horizonal dashed line represents the outage probability $P_{\text {out }}$ of Eq. (18). Concerning the CCDF curves, the flatter the curve is the better the system performs.

To fulfill the quality criterion of Eq. (18) for each case there exists an upper limit of the system load $\frac{K}{K_{\mathrm{B}}}$, which may be smaller than the full load $\frac{K}{K_{\mathrm{B}}}$ equal to one. At the given values of $T_{\mathrm{dBm}}$ and $F_{\mathrm{dB}}$ the SA-based JOINT shows advantages over the cellular system in the interference elimination. Moreover, the larger the SA size $K_{\mathrm{B}}$ or the larger the reuse factor $r$ becomes, the greater performance improvement can be obtained.

\subsection{Spectrum efficiency}

Based on the analysis in Sects. 5.2 and 5.3 it is seen that the improved performance of JOINT can be obtained by increasing the SA size $K_{\mathrm{B}}$, increasing the reuse factor $r$ or reducing the system load $\frac{K}{K_{\mathrm{B}}}$, at the sacrifice of spectrum efficiency. To achieve a high spectrum efficiency is a challenge for the future mobile radio systems. A trade-off between the performance and the spectrum efficiency has to be made for the system design. In conventional cellular system spectrum efficiency $\eta_{\mathrm{c}}$ is defined as the ratio of the total data rate per cell and the corresponding bandwidth used (Steele, 1992; David and Benkner, 1996). To adapt the definition to the
SA concept it is fair to consider the spectrum efficiency per AP. With the system load $\frac{K}{K_{\mathrm{B}}}$ fulfilling the quality criterion of Eq. (18) and the corresponding reuse factor $r$, with the data rate $R_{\mathrm{d}}$ equal to $62.5 \mathrm{kBits} / \mathrm{s}$ per MT per subcarrier and with the bandwidth $B$ equal to the subcarrier spacing $39.063 \mathrm{kHz}$, the spectrum efficiency

$\eta_{\mathrm{c}}=\frac{R_{\mathrm{d}} \frac{K}{K_{\mathrm{B}}}}{B r}$

is defined based on the analysis of the BER statistics in Sect. 5.3.

According to Eq. (19) the spectrum efficiencies $\eta_{\mathrm{c}}$ are calculated for different combinations of the SA size $K_{\mathrm{B}}$, the number $K$ of MTs per SA and the reuse factor $r$. The corresponding results are listed in Table 2 . The symbol - means that the corresponding case does not fulfill the quality of service (QoS) criterion of Eq. (18). The optimum value of the spectrum efficiency $\eta_{\mathrm{c}}$ is the maximum of the spectrum efficiencies $\eta_{\mathrm{c}}$ of Eq. (19) for each case of $K_{\mathrm{B}}$, as highlighted in Table 2. It can be seen that the SA-based JOINT has great potentials in improving the spectrum efficiency $\eta_{\mathrm{c}}$ compared to the conventional cellular system, whose optimum value of the spectrum efficiency $\eta_{\mathrm{c}}$ is equal to $0.1333 \mathrm{bits} / \mathrm{s} / \mathrm{Hz}$ as shown in Table 1.

\section{Conclusions}

In the present paper the average BER performance and the BER statistics of the uplink of JOINT are investigated in the given multi-SA scenario. The influence of parameters like, e.g., the SA size $K_{\mathrm{B}}$, the reuse factor $r$ and the system load $\frac{K}{K_{\mathrm{B}}}$ on the system performance is studied. SA-based JOINT shows a great potential of interference elimination which improves the system performance and enhances the spectrum efficiency as compared to the conventional cellular system. 
Acknowledgements. The authors gratefully appreciate the stimulating discussions and the fruitful exchange of ideas with P. W. Baier and the co-workers at the Research Group for RF Communications, University of Kaiserslautern and the colleagues at Siemens AG.

\section{References}

Boejeson, H., Bergljung, C., and Olsson, L. G.: Outdoor microcell measurements at $1700 \mathrm{MHz}$, Proc. of the 42nd IEEE Vehicular Technology Conference (VTC'92), Denver, 929-931, 1992.

Couch, L. W.: Digital and Analog Communication Systems, 5th Edition, Prentice-Hall International, 1997.

David, K. and Benkner, T.: Digitale Mobilfunksysteme, Stuttgart, B. G. Teubner, 1996.

Klein, A.: Multiuser detection of CDMA signals - algorithms and their applications to cellular mobile radio systems, Fortschrittberichte VDI, Reihe 10, Nr. 423, Düsseldorf, VDI Verlag, 1996. van Nee, R. D. J. and Prasad, R.: OFDM for Wireless Multimedia Communications, Boston, Artech House, 2000.

Steil, R.: Mobile Radio Communications, London, Pentech Press, 1992.

Steil, A.: Spektrale Effizienz digitaler zellularer CDMAMobilfunksysteme mit gemeinsamer Detection, Fortschrittberichte VDI, Reihe 10, Nr. 437, Düsseldorf, VDI Verlag, 1995.

Verdu, S.: Multiuser Detection, Cambridge, Cambridge University Press, 1998.

Weber, T., Maniatis, I., Sklavos, A., and Liu, Y.: Joint transmission and detection integrated network (JOINT), a generic proposal for beyond $3 \mathrm{G}$ systems, Proc. of the 9th International Conference on Telecommunications (ICT'02), Beijing, vol. 3, 479-483, 2002.

Xia, H. H., Bertoni, H. L., and Macial, L. R.: Radio propagation measurements and modeling for line-of-sight microcellular systems, Proc. of the 42nd IEEE Vehicular Technology Conference (VTC'92), Denver, 349-354., 1992. 